\title{
How to Leak a Secret
}

\author{
Ronald L. Rivest ${ }^{1}$, Adi Shamir ${ }^{2}$, and Yael Tauman ${ }^{2}$ \\ 1 Laboratory for Computer Science, Massachusetts Institute of Technology, \\ Cambridge, MA 02139, rivest@mit.edu \\ 2 Computer Science department, The Weizmann Institute, Rehovot 76100, Israel. \\ \{shamir, tauman\}@wisdom.weizmann.ac.il
}

\begin{abstract}
In this paper we formalize the notion of a ring signature, which makes it possible to specify a set of possible signers without revealing which member actually produced the signature. Unlike group signatures, ring signatures have no group managers, no setup procedures, no revocation procedures, and no coordination: any user can choose any set of possible signers that includes himself, and sign any message by using his secret key and the others' public keys, without getting their approval or assistance. Ring signatures provide an elegant way to leak authoritative secrets in an anonymous way, to sign casual email in a way which can only be verified by its intended recipient, and to solve other problems in multiparty computations. The main contribution of this paper is a new construction of such signatures which is unconditionally signer-ambiguous, provably secure in the random oracle model, and exceptionally efficient: adding each ring member increases the cost of signing or verifying by a single modular multiplication and a single symmetric encryption.
\end{abstract}

Keywords: signature scheme, ring signature scheme, signer-ambiguous signature scheme, group signature scheme, designated verifier signature scheme.

\section{Introduction}

The general notion of a group signature scheme was introduced in 1991 by Chaum and van Heyst 2. In such a scheme, a trusted group manager predefines certain groups of users and distributes specially designed keys to their members. Individual members can then use these keys to anonymously sign messages on behalf of their group. The signatures produced by different group members look indistinguishable to their verifiers, but not to the group manager who can revoke the anonymity of misbehaving signers.

In this paper we formalize the related notion of ring signature schemes. These are simplified group signature schemes which have only users and no managers (we call such signatures "ring signatures" instead of "group signatures" since rings are geometric regions with uniform periphery and no center). Group signatures are useful when the members want to cooperate, while ring signatures are useful when the members do not want to cooperate. Both group signatures and

C. Boyd (Ed.): ASIACRYPT 2001, LNCS 2248, pp. 552565, 2001.

(C) Springer-Verlag Berlin Heidelberg 2001 
ring signatures are signer-ambiguous, but in a ring signature scheme there are no prearranged groups of users, there are no procedures for setting, changing, or deleting groups, there is no way to distribute specialized keys, and there is no way to revoke the anonymity of the actual signer (unless he decides to expose himself). Our only assumption is that each member is already associated with the public key of some standard signature scheme such as RSA. To produce a ring signature, the actual signer declares an arbitrary set of possible signers that includes himself, and computes the signature entirely by himself using only his secret key and the others' public keys. In particular, the other possible signers could have chosen their RSA keys only in order to conduct e-commerce over the internet, and may be completely unaware that their public keys are used by a stranger to produce such a ring signature on a message they have never seen and would not wish to sign.

The notion of ring signatures is not completely new, but previous references do not crisply formalize the notion, and propose constructions that are less efficient and/or that have different, albeit related, objectives. They tend to describe this notion in the context of general group signatures or multiparty constructions, which are quite inefficient. For example, Chaum et al. [2]'s schemes three and four, and the two signature schemes in Definitions 2 and 3 of Camenisch's paper 1] can be viewed as ring signature schemes. However the former schemes require zero-knowledge proofs with each signature, and the latter schemes require as many modular exponentiations as there are members in the ring. Cramer et al. 3. shows how to produce witness-indistinguishable interactive proofs. Such proofs could be combined with the Fiat-Shamir technique to produce ring signature schemes. Similarly, DeSantis et al. [10] show that interactive SZK for random self-reducible languages are closed under monotone boolean operations, and show the applicability of this result to the construction of a ring signature scheme (although they don't use this terminology).

The direct construction of ring signatures proposed in this paper is based on a completely different idea, and is exceptionally efficient for large rings (adding only one modular multiplication and one symmetric encryption per ring member both to generate and to verify such signatures). The resultant signatures are unconditionally signer-ambiguous and provably secure in the random oracle model.

\section{Definitions and Applications}

\subsection{Ring Signatures}

Terminology: We call a set of possible signers a ring. We call the ring member who produces the actual signature the signer and each of the other ring members a non-signer.

We assume that each possible signer is associated (via a PKI directory or certificate) with a public key $P_{k}$ that defines his signature scheme and specifies his verification key. The corresponding secret key (which is used to generate regular signatures) is denoted by $S_{k}$. The general notion of a ring signature scheme 
does not require any special properties of these individual signing schemes, but our simplest construction assumes that they use trapdoor one-way permutations (such as the RSA functions) to generate and verify signatures.

A ring signature scheme is defined by two procedures:

- ring-sign $\left(m, P_{1}, P_{2}, \ldots, P_{r}, s, S_{s}\right)$ which produces a ring signature $\sigma$ for the message $m$, given the public keys $P_{1}, P_{2}, \ldots, P_{r}$ of the $r$ ring members, together with the secret key $S_{s}$ of the $s$-th member (who is the actual signer).

- ring-verify $(m, \sigma)$ which accepts a message $m$ and a signature $\sigma$ (which includes the public keys of all the possible signers), and outputs either true or false.

A ring signature scheme is set-up free: The signer does not need the knowledge, consent, or assistance of the other ring members to put them in the ring - all he needs is knowledge of their regular public keys. Different members can use different independent public key signature schemes, with different key and signature sizes. Verification must satisfy the usual soundness and completeness conditions, but in addition we want the signatures to be signer-ambiguous in the sense that the verifier should be unable to determine the identity of the actual signer in a ring of size $r$ with probability greater than $1 / r$. This limited anonymity can be either computational or unconditional. Our main construction provides unconditional anonymity in the sense that even an infinitely powerful adversary with access to an unbounded number of chosen-message signatures produced by the same ring member cannot guess his identity with any advantage, and cannot link additional signatures to the same signer.

\section{$2.2 \quad$ Leaking Secrets}

To motivate the title for this paper, suppose that Bob (also known as "Deep Throat") is a member of the cabinet of Lower Kryptonia, and that Bob wishes to leak a juicy fact to a journalist about the escapades of the Prime Minister, in such a way that Bob remains anonymous, yet such that the journalist is convinced that the leak was indeed from a cabinet member.

Bob cannot send to the journalist a standard digitally signed message, since such a message, although it convinces the journalist that it came from a cabinet member, does so by directly revealing Bob's identity.

It also doesn't work for Bob to send the journalist a message through a standard anonymizer, since the anonymizer strips off all source identification and authentication: the journalist would have no reason to believe that the message really came from a cabinet member at all.

A standard group signature scheme does not solve the problem, since it requires the prior cooperation of the other group members to set up, and leaves Bob vulnerable to later identification by the group manager, who may be controlled by the Prime Minister.

The correct approach is for Bob to send the story to the journalist through an anonymizer, signed with a ring signature scheme that names each cabinet 
member (including himself) as a ring member. The journalist can verify the ring signature on the message, and learn that it definitely came from a cabinet member. He can even post the ring signature in his paper or web page, to prove to his readers that the juicy story came from a reputable source. However, neither he nor his readers can determine the actual source of the leak, and thus the whistleblower has perfect protection even if the journalist is later forced by a judge to reveal his "source" (the signed document).

\subsection{Designated Verifier Signature Schemes}

A designated verifier signature scheme is a signature scheme in which signatures can only be verified by a single "designated verifier" chosen by the signer. This concept was first introduced by Jakobsson Sako and Impagliazzo at Eurocrypt 96 [6]. A typical application is to enable users to authenticate casual emails without being legally bound to their contents. For example, two companies may exchange drafts of proposed contracts. They wish to add to each email an authenticator, but not a real signature which can be shown to a third party (immediately or years later) as proof that a particular draft was proposed by the other company. A designated verifier scheme can thus be viewed as a "light signature scheme" which can authenticate messages to their intended recipients without having the nonrepudiation property.

One approach would be to use zero knowledge interactive proofs, which can only convince their verifiers. However, this requires interaction and is difficult to integrate with standard email systems and anonymizers. We can use noninteractive zero knowledge proofs, but then the authenticators become signatures which can be shown to third parties. Another approach is to agree on a shared secret symmetric key $k$, and to authenticate each contract draft by appending a message authentication code (MAC) for the draft computed with key $k$. A third party would have to be shown the secret key to validate a MAC, and even then he wouldn't know which of the two companies computed the MAC. However, this requires an initial set-up procedure, in which we still face the problem of authenticating the emailed choice of $k$ without actually signing it.

A designated verifier scheme provides a simple solution to this problem: company A can sign each draft it sends, naming company B as the designated verifier. This can be easily achieved by using a ring signature scheme with companies A and $\mathrm{B}$ as the ring members. Just as with a MAC, company B knows that the message came from company A (since no third party could have produced this ring signature), but company B cannot prove to anyone else that the draft of the contract was signed by company A, since company B could have produced this draft by itself. Unlike the case of MAC's, this scheme uses public key cryptography, and thus A can send unsolicited email to B signed with the ring signature without any preparations, interactions, or secret key exchanges. By using our proposed ring signature scheme, we can turn standard signature schemes into designated verifier schemes which can be added at almost no cost as an extra option to any email system. 


\subsection{Efficiency of Our Ring Signature Scheme}

When based on Rabin or RSA signatures, our ring signature scheme is particularly efficient:

- signing requires one modular exponentiation, plus one or two modular multiplications for each non-signer.

- verification requires one or two modular multiplications for each ring member.

In essence, generating or verifying a ring signature costs the same as generating or verifying a regular signature plus an extra multiplication or two for each non-signer, and thus the scheme is truly practical even when the ring contains hundreds of members. It is two to three orders of magnitude faster than Camenisch's scheme, whose claimed efficiency is based on the fact that it is 4 times faster than earlier known schemes (see bottom of page 476 in his paper 11). In addition, a Camenisch-like scheme uses linear algebra in the exponents, and thus requires all the members to use the same prime modulus $p$ in their individual signature schemes. One of our design criteria is that the signer should be able to assemble an arbitrary ring without any coordination with the other ring members. In reality, if one wants to use other users' public keys, they are much more likely to be RSA keys, and even if they are based on discrete logs, different users are likely to have different moduli $p$. The only realistic way to arrange a Camenisch-like signature scheme is thus to have a group of consenting parties.

Note that the size of any ring signature must grow linearly with the size of the ring, since it must list the ring members; this is an inherent disadvantage of ring signatures as compared to group signatures that use predefined groups.

\section{The Proposed Ring Signature Scheme (RSA Version)}

Suppose that Alice wishes to sign a message $m$ with a ring signature for the ring of $r$ individuals $A_{1}, A_{2}, \ldots, A_{r}$, where the signer Alice is $A_{s}$, for some value of $s, 1 \leq s \leq r$. To simplify the presentation and proof, we first describe a ring signature scheme in which all the ring members use RSA [9] as their individual signature schemes. The same construction can be used for any other trapdoor one way permutation, but we have to modify it slightly in order to use trapdoor one way functions (as in, for example, Rabin's signature scheme [8]).

\subsection{RSA Trap-Door Permutations}

Each ring member $A_{i}$ has an RSA public key $P_{i}=\left(n_{i}, e_{i}\right)$ which specifies the trapdoor one-way permutation $f_{i}$ of $\mathbf{Z}_{n_{i}}$ :

$$
f_{i}(x)=x^{e_{i}} \quad\left(\bmod n_{i}\right) .
$$

We assume that only $A_{i}$ knows how to compute the inverse permutation $f_{i}^{-1}$ efficiently, using trap-door information; this is the original Diffie-Hellman model [4] for public-key cryptography. 


\section{Extending trap-door permutations to a common domain}

The trap-door RSA permutations of the various ring members will have domains of different sizes (even if all the moduli $n_{i}$ have the same number of bits). This makes it awkward to combine the individual signatures, and thus we extend all the trap-door permutations to have as their common domain the same set $\{0,1\}^{b}$, where $2^{b}$ is some power of two which is larger than all the moduli $n_{i}$ 's.

For each trap-door permutation $f_{i}$ over $\mathbf{Z}_{n_{i}}$, we define the extended trap-door permutation $g_{i}$ over $\{0,1\}^{b}$ in the following way. For any $b$-bit input $m$ define nonnegative integers $q_{i}$ and $r_{i}$ so that $m=q_{i} n_{i}+r_{i}$ and $0 \leq r_{i}<n_{i}$. Then

$$
g_{i}(m)= \begin{cases}q_{i} n_{i}+f_{i}\left(r_{i}\right) & \text { if }\left(q_{i}+1\right) n_{i} \leq 2^{b} \\ m & \text { else. }\end{cases}
$$

Intuitively, $g_{i}$ is defined by using $f_{i}$ to operate on the low-order digit of the $n_{i}$-ary representation of $m$, leaving the higher order digits unchanged. The exception is when this might cause a result larger than $2^{b}-1$, in which case $m$ is unchanged. If we choose a sufficiently large $b$ (e.g. 160 bits larger than any of the $n_{i}$ ), the chance that a randomly chosen $m$ is unchanged by the extended $g_{i}$ becomes negligible. (A stonger but more expensive approach, which we don't need, would use instead of $g_{i}(m)$ the function $g_{i}^{\prime}(m)=g_{i}\left(\left(2^{b}-1\right)-g_{i}(m)\right)$ which can modify all its inputs). The function $g_{i}$ is clearly a permutation over $\{0,1\}^{b}$, and it is a one-way trap-door permutation since only someone who knows how to invert $f_{i}$ can invert $g_{i}$ efficiently on more than a negligible fraction of the possible inputs.

\subsection{Symmetric Encryption}

We assume the existence of a publicly defined symmetric encryption algorithm $E$ such that for any key $k$ of length $l$, the function $E_{k}$ is a permutation over $b$-bit strings. Here we use the random (permutation) oracle model which assumes that all the parties have access to an oracle that provides truly random answers to new queries of the form $E_{k}(x)$ and $E_{k}^{-1}(y)$, provided only that they are consistent with previous answers and with the requirement that $E_{k}$ be a permutation (e.g. see [7]).

\subsection{Hash Functions}

We assume the existence of a publicly defined collision-resistant hash function $h$ that maps arbitrary inputs to strings of length $l$, which are used as keys for $E$. We model $h$ as a random oracle. (Since $h$ need not be a permutation, different queries may have the same answer, and we will disallow " $h^{-1}$ " queries.)

\subsection{Combining Functions}

We define a family of keyed "combining functions" $C_{k, v}\left(y_{1}, y_{2}, \ldots, y_{r}\right)$ which take as input a key $k$, an initialization value $v$, and arbitrary values $y_{1}, y_{2}, \ldots$, $y_{r}$ in $\{0,1\}^{b}$. Each such combining function uses $E_{k}$ as a sub-procedure, and 
produces as output a value $z$ in $\{0,1\}^{b}$ such that given any fixed values for $k$ and $v$, we have the following properties.

1. Permutation on each input: For each $s, 1 \leq s \leq r$, and for any fixed values of all the other inputs $y_{i}, i \neq s$, the function $C_{k, v}$ is a one-to-one mapping from $y_{s}$ to the output $z$.

2. Efficiently solvable for any single input: For each $s, 1 \leq s \leq r$, given a $b$-bit value $z$ and values for all inputs $y_{i}$ except $y_{s}$, it is possible to efficiently find a $b$-bit value for $y_{s}$ such that $C_{k, v}\left(y_{1}, y_{2}, \ldots, y_{r}\right)=z$.

3. Infeasible to solve verification equation for all inputs without trap-doors: Given $k, v$, and $z$, it is infeasible for an adversary to solve the equation

$$
C_{k, v}\left(g_{1}\left(x_{1}\right), g_{2}\left(x_{2}\right), \ldots, g_{r}\left(x_{r}\right)\right)=z
$$

for $x_{1}, x_{2}, \ldots, x_{r}$, (given access to each $g_{i}$, and to $E_{k}$ ) if the adversary can't invert any of the trap-door functions $g_{1}, g_{2}, \ldots, g_{r}$.

For example, the function

$$
C_{k, v}\left(y_{1}, y_{2}, \ldots, y_{r}\right)=y_{1} \oplus y_{2} \oplus \cdots \oplus y_{r}
$$

(where $\oplus$ is the exclusive-or operation on $b$-bit words) satisfies the first two of the above conditions, and can be kept in mind as a candidate combining function. Indeed, it was the first one we tried. But it fails the third condition since for any choice of trapdoor one-way permutations $g_{i}$, it is possible to use linear algebra when $r$ is large enough to find a solution for $x_{1}, x_{2}, \ldots, x_{r}$ without inverting any of the $g_{i}$ 's. The basic idea of the attack is to choose a random value for each $x_{i}$, and to compute each $y_{i}=g_{i}\left(x_{i}\right)$ in the easy forward direction. If the number of values $r$ exceeds the number of bits $b$, we can find with high probability a subset of the $y_{i}$ bit strings whose XOR is any desired $b$-bit target $z$. However, our goal is to represent $z$ as the XOR of all the values $y_{1}, y_{2}, \ldots, y_{r}$ rather than as a XOR of a random subset of these values. To overcome this problem, we choose for each $i$ two random values $x_{i}^{\prime}$ and $x_{i}^{\prime \prime}$, and compute their corresponding $y_{i}^{\prime}=g_{i}\left(x_{i}^{\prime}\right)$ and $y_{i}^{\prime \prime}=g_{i}\left(x_{i}^{\prime \prime}\right)$. We then define for each $i y_{i}^{\prime \prime \prime}=y_{i}^{\prime} \oplus y_{i}^{\prime \prime}$, and modify the target value to $z^{\prime}=z \oplus y_{1}^{\prime} \oplus y_{2}^{\prime}, \ldots \oplus y_{r}^{\prime}$. We use the previous algorithm to represent $z^{\prime}$ as a XOR of a random subset of $y_{i}^{\prime \prime \prime}$ values. After simplification, we get a representation of the original $z$ as the XOR of a set of $r$ values, with exactly one value chosen from each pair $\left(y_{i}^{\prime}, y_{i}^{\prime \prime}\right)$. By choosing the corresponding value of either $x_{i}^{\prime}$ or $x_{i}^{\prime \prime}$, we can solve the verification equation without inverting any of the trapdoor one-way permutations $g_{i}$. (One approach to countering this attack, which we don't explore further here, is to let $b$ grow with $r$.)

Even worse problems can be shown to exist in other natural combining functions such as addition mod $2^{b}$. Assume that we use the RSA trapdoor functions $g_{i}\left(x_{i}\right)=x_{i}^{3}\left(\bmod n_{i}\right)$ where all the moduli $n_{i}$ have the same size $b$. It is known [5] that any nonnegative integer $\mathrm{z}$ can be efficiently represented as the sum of exactly nine nonnegative integer cubes $x_{1}^{3}+x_{2}^{3}+\ldots+x_{9}^{3}$. If $z$ is a $b$-bit target value, we can expect each one of the $x_{i}^{3}$ to be slightly shorter than $z$, and thus their values are not likely to be affected by reducing each $x_{i}^{3}$ modulo 
the corresponding $b$-bit $n_{i}$. Consequently, we can solve the verification equation $\left(x_{1}^{3} \bmod n_{1}\right)+\left(x_{2}^{3} \bmod n_{2}\right) \ldots+\left(x_{9}^{3} \bmod n_{9}\right)=z\left(\bmod 2^{b}\right)$ with nine RSA permutations without inverting any one of them.

Our proposed combining function utilizes the symmetric encryption function $E_{k}$ as follows:

$C_{k, v}\left(y_{1}, y_{2}, \ldots, y_{r}\right)=E_{k}\left(y_{r} \oplus E_{k}\left(y_{r-1} \oplus E_{k}\left(y_{r-2} \oplus E_{k}\left(\ldots \oplus E_{k}\left(y_{1} \oplus v\right) \ldots\right)\right)\right)\right)$.

This function is applied to the sequence $\left(y_{1}, y_{2}, \ldots, y_{r}\right)$, where $y_{i}=g_{i}\left(x_{i}\right)$, as shown in Figure 1; the resulting function is provably secure in the random oracle model.

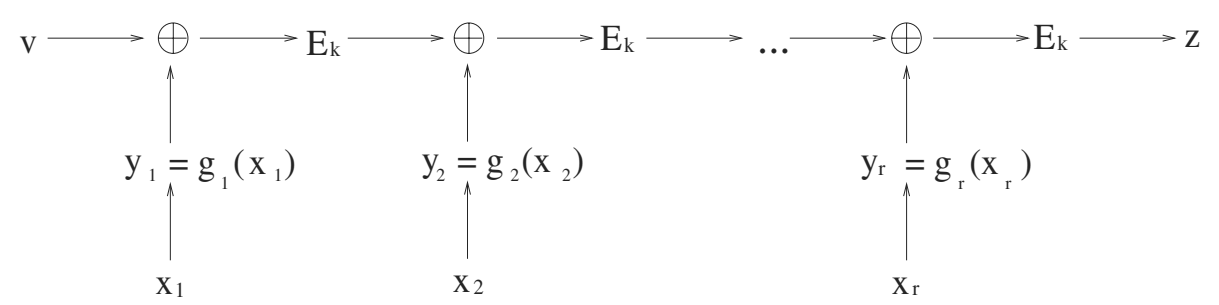

Fig. 1. An illustration of the proposed combining function

It is clearly a permutation on each input, since the XOR, $g_{i}$, and $E_{k}$ functions are permutations. In addition, it is efficiently solvable for any single input since knowledge of $k$ makes it possible to run the evaluation forwards from the initial $v$ and backwards from the final $z$ in order to uniquely compute any missing value $y_{i}$. This function can be used to verify signatures by using a hashed version of $m$ to choose the symmetric key $k$, and forcing the output $z$ to be equal to the input $v$. This consistency condition $C_{k, v}\left(y_{1}, y_{2}, \ldots, y_{r}\right)=v$ bends the line into the ring shape shown in Fig. 2.

A slightly more compact ring signature variant can be obtained by always selecting 0 as the "glue value" $v$. This variant is also secure, but we prefer the total ring symmetry of our main proposal.

We now formally describe the signature generation and verification procedures:

\section{Generating a ring signature:}

Given the message $m$ to be signed, his secret key $S_{s}$, and the sequence of public keys $P_{1}, P_{2}, \ldots, P_{r}$ of all the ring members, the signer computes a ring signature as follows.

1. Choose a key: The signer first computes the symmetric key $k$ as the hash of the message $m$ to be signed:

$$
k=h(m)
$$

(a more complicated variant computes $k$ as $h\left(m, P_{1}, \ldots, P_{r}\right)$; however, the simpler construction is also secure.) 


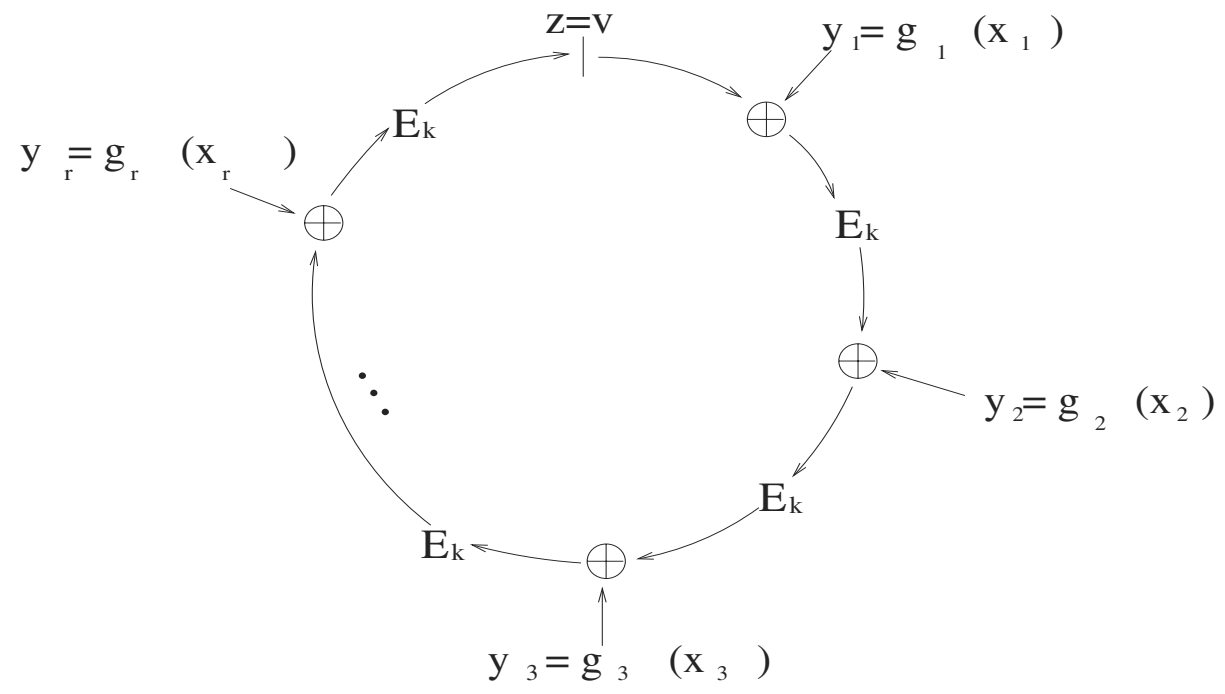

Fig. 2. Ring signatures

2. Pick a random glue value: Second, the signer picks an initialization (or "glue") value $v$ uniformly at random from $\{0,1\}^{b}$.

3. Pick random $x_{i}$ 's: Third, the signer picks random $x_{i}$ for all the other ring members $1 \leq i \leq r, i \neq s$ uniformly and independently from $\{0,1\}^{b}$, and computes

$$
y_{i}=g_{i}\left(x_{i}\right)
$$

4. Solve for $y_{s}$ : Fourth, the signer solves the following ring equation for $y_{s}$ :

$$
C_{k, v}\left(y_{1}, y_{2}, \ldots, y_{r}\right)=v \text {. }
$$

By assumption, given arbitrary values for the other inputs, there is a unique value for $y_{s}$ satisfying the equation, which can be computed efficiently.

5. Invert the signer's trap-door permutation: Fifth, the signer uses his knowledge of his trapdoor in order to invert $g_{s}$ on $y_{s}$ to obtain $x_{s}$ :

$$
x_{s}=g_{s}^{-1}\left(y_{s}\right) \text {. }
$$

6. Output the ring signature: The signature on the message $m$ is defined to be the $(2 r+1)$-tuple:

$$
\left(P_{1}, P_{2}, \ldots, P_{r} ; v ; x_{1}, x_{2}, \ldots, x_{r}\right) .
$$

\section{Verifying a ring signature:}

A verifier can verify an alleged signature

$$
\left(P_{1}, P_{2}, \ldots, P_{r} ; v ; x_{1}, x_{2}, \ldots, x_{r}\right) .
$$

on the message $m$ as follows. 
1. Apply the trap-door permutations: First, for $i=1,2, \ldots, r$ the verifier computes

$$
y_{i}=g_{i}\left(x_{i}\right)
$$

2. Obtain $k$ : Second, the verifier hashes the message to compute the encryption key $k$ :

$$
k=h(m) .
$$

3. Verify the ring equation: Finally, the verifier checks that the $y_{i}$ 's satisfy the fundamental equation:

$$
C_{k, v}\left(y_{1}, y_{2}, \ldots, y_{r}\right)=v \text {. }
$$

If the ring equation (2) is satisfied, the verifier accepts the signature as valid. Otherwise the verifier rejects.

\subsection{Security}

The identity of the signer is unconditionally protected with our ring signature scheme. To see this, note that for each $k$ and $v$ the ring equation has exactly $\left(2^{b}\right)^{(r-1)}$ solutions, and all of them can be chosen by the signature generation procedure with equal probability, regardless of the signer's identity. This argument does not depend on any complexity-theoretic assumptions or on the randomness of the oracle.

The soundness of the ring signature scheme must be computational, since ring signatures cannot be stronger than the individual signature scheme used by the possible signers. Our goal now is to show that in the random oracle model, any forging algorithm $A$ which can generate with non-negligible probability a new ring signature for $m$ by analysing polynomially many ring signatures for other chosen messages $m_{j} \neq m$, can be turned into an algorithm $B$ which inverts one of the trapdoor one-way functions $g_{i}$ on random inputs $y$ with non-negligible probability.

Algorithm $A$ accepts the public keys $P_{1}, P_{2}, \ldots, P_{r}$ (but not any of the corresponding secret keys) and is given oracle access to $h, E, E^{-1}$, and to a ring signing oracle. It can work adaptively, querying the oracles at arguments that may depend on previous answers. Eventually, it must produce a valid ring signature on a new message that was not presented to the signing oracle, with a non-negligible probability (over the random answers of the oracles and its own random tape).

Algorithm $B$ uses algorithm $A$ as a black box, but has full control over its oracles. $A$ must query the oracle about all the symmetric encryptions along the forged ring signature of $m$ (otherwise the probability of satisfying the ring equation becomes negligible). Without loss of generality, we can assume that each one of these $r$ symmetric encryptions is queried once either in the "clockwise" $E_{k}$ direction or in the "counterclockwise" $E_{k}^{-1}$ direction, but not in both directions since this is redundant. When $A$ makes its polynomially many querries of $E_{k}$ and $E_{k}^{-1}$ with various keys $k=h(m), B$ can guess which $k$ will be involved in the 
actual forgery with non-negligible probability, but it cannot guess which subset of $r$ queries will be used in the final forgery and in which order they will occur along the satisfied ring equation since there are too many possibilities.

Algorithm $B$ can easily simulate the ring signing oracle for all the other $m_{j}$ by providing random vectors $\left(v, x_{1}, x_{2}, \ldots, x_{r}\right)$ as their ring signatures, and adjusting the random answers for queries of the form $E_{h\left(m_{j}\right)}$ and $E_{h\left(m_{j}\right)}^{-1}$ to support the correctness of the ring equation for these messages. Note that $A$ cannot ask relevant oracle questions which will limit $B$ 's freedom of choice before providing $m_{j}$ to the signing oracle since all the values along the actual ring signature (including $v$ ) are chosen randomly by $B$ when it provides the requested signature, and cannot be guessed in advance by $A$. In addition, we use the assumption that $h$ is collision resistant to show that $E$ and $E^{-1}$ queries with key $k_{j}=h\left(m_{j}\right)$ will not constrain the answers to $E$ and $E^{-1}$ queries with key $k=h(m)$ which will be used in the final forgery, since they use different keys.

The goal of algorithm $B$ is to compute for some $i x_{i}=g_{i}^{-1}(y)$ for random inputs $y$ 's with non-negligible probability. This will reduce the security of the ring signature to the security of the individual signature schemes. The basic idea of the reduction is to slip this random $y$ as the "gap" between the output and input values of two cyclically consecutive $E$ 's along the ring equation of the final forgery, which forces $A$ to close the gap by providing the corresponding $x_{i}$ in the generated signature. Note that $y$ is a random value which is known to $B$ but not to $A$, and thus $A$ cannot "recognize the trap" and refuse to sign the corresponding messages.

The main difficuly is that $A$ can close gaps between $E$ values not only by inverting trapdoor one-way functions, but also by evaluating these functions in the easy forward direction (as done by the real signer in the generation of ring signatures). To overcome this difficulty, we note that in any valid ring signature produced by $A$, there must be a gap somewhere between two cyclically consecutive occurences of $E$ in which the queries were computed in one of the following three ways:

- The oracle for the $i$-th $E$ was queried in the "clockwise" direction and the oracle for the $i+1$-st $E$ was queried in the "counterclockwise" direction.

- Both $E$ 's were queried in the "clockwise" direction, but the $i$-th $E$ was queried after the $i+1$-st $E$.

- Both $E$ 's were queried in the "counterclockwise" direction, but the $i$-th $E$ was queried before the $i+1$-st $E$.

In all these cases, $B$ can provide a random answer to the later query which is based on his knowledge of input and output of the earlier query in such a way that the XOR of the values acros the gap is the desired $y$. This will force $A$ to compute the corresponding $g_{i}^{-1}(y)$ in order to fill in this gap in its final ring signature.

$B$ does not know which queries will be these cyclically consecutive queries in the forged ring signature, and thus he has to guess their identity. However, he has to make only two guesses and thus the probability of guessing correctly is $1 / Q^{2}$ 
where $Q$ is the total number of queries made by the forger $A$. Consequently, $B$ will manage to compute $g_{i}^{-1}(y)$ for a random $y$ and some $i$ with non-negligible probability.

When the trapdoor one-way functions $g_{i}$ are RSA functions, we can slightly strengthen the result. Since RSA is homomorphic, we can randomize $y$ by computing $y^{\prime}=y * t^{e_{i}}\left(\bmod n_{i}\right)$ for a randomly chosen $t$. By using $y^{\prime}$ instead of $y$, we can show that successful forgeries of ring signatures can be used to extract modular roots from particular numbers such as $y=2$, and not just from random inputs $y$. This is not necessarily true for other trapdoor functions, since the forger $A$ can intentionally decide not to produce any forgeries in which one of the gaps between cyclically consecutive $E$ functions happens to be 2 .

\section{Our Ring Signature Scheme (Rabin Version)}

Rabin's public-key cryptosystem [8] has more efficient signature verification than RSA, since verification involves squaring rather than cubing, which reduces the number of modular multiplications from 2 to 1 . However, we need to deal with the fact that the Rabin mapping $f_{i}\left(x_{i}\right)=x_{i}^{2}\left(\bmod n_{i}\right)$ is not a permutation over $\mathbf{Z}_{n_{i}}^{*}$, and thus only one quarter of the messages can be signed, and those which can be signed have multiple signatures.

The operational fix is the natural one: when signing, change your last random choice of $x_{s-1}$ if $g_{s}^{-1}\left(y_{s}\right)$ is undefined. Since only one trapdoor one-way function has to be inverted, the signer should expect on average to try four times before succeeding in producing a ring signature. The complexity of this search is essentially the same as in the case of regular Rabin signatures, regardless of the size of the ring.

A more important difference is in the proof of unconditional anonymity, which relied on the fact that all the mappings were permutations. When the $g_{i}$ are not permutations, there can be noticable differences between the distribution of randomly chosen and computed $x_{i}$ values in given ring signatures. This could lead to the identification of the real signer among all the possible signers, and can be demonstrated to be a real problem in many concrete types of trapdoor one-way functions.

We overcome this difficulty in the case of Rabin signatures with the following simple observation:

Theorem 1. Let $S$ be a given finite set of "marbles" and let $B_{1}, B_{2}, \ldots, B_{n}$ be disjoint subsets of $S$ (called "buckets") such that all non-empty buckets have the same number of marbles, and every marble in $S$ is in exactly one bucket. Consider the following sampling procedure: pick a bucket at random until you find a non-empty bucket, and then pick a marble at random from that bucket. Then this procedure picks marbles from $S$ with uniform probability distribution.

Proof. Trivial.

Rabin's functions $f_{i}\left(x_{i}\right)=x_{i}^{2}\left(\bmod n_{i}\right)$ are extended to functions $g_{i}\left(x_{i}\right)$ over $\{0,1\}^{b}$ in the usual way. Both the marbles and the buckets are all the $b$-bit 
numbers $u=q_{i} n_{i}+r_{i}$ in which $r_{i} \in \mathbf{Z}_{n_{i}}^{*}$ and $\left(q_{i}+1\right) n_{i} \leq 2^{b}$ Each marble is placed in the bucket to which it is mapped by the extended Rabin mapping $g_{i}$. We know that each bucket contains either zero or four marbles, and the lemma inplies that the sampled distribution of the marbles $x_{i}$ is exactly the same regardless of whether they were chosen at random or picked at random among the computed inverses in a randomly chosen bucket. Consequently, even an infinitely powerful adversary cannot distinguish between signers and nonsigners by analysing actual ring signatures produced by one of the possible signers.

\section{$5 \quad$ Generalizations and Special Cases}

The notion of ring signatures has many interesting extensions and special cases. In particular, ring signatures with $r=1$ can be viewed as a randomized version of Rabin's signature scheme: As shown in Fig. 3, the verification condition can be written as $\left(x^{2} \bmod n\right)=v \oplus E_{h(m)}^{-1}(v)$. The right hand side is essentially a hash of the message $m$, randomized by the choice of $v$.

Ring signatures with $r=2$ have the ring equation:

$$
E_{h(m)}\left(x_{2}^{2} \oplus E_{h(m)}\left(x_{1}^{2} \oplus v\right)\right)=v
$$

(see Fig. 3). A simpler ring equation (which is not equivalent but has the same security properties) is:

$$
\left(x_{1}^{2} \bmod n_{1}\right)=E_{h(m)}\left(x_{2}^{2} \bmod n_{2}\right)
$$

where the modular squares are extended to $\{0,1\}^{b}$ in the usual way. This is our recommended method for implementing designated verifier signatures in email systems, where $n_{1}$ is the public key of the sender and $n_{2}$ is the public key of the recipient.
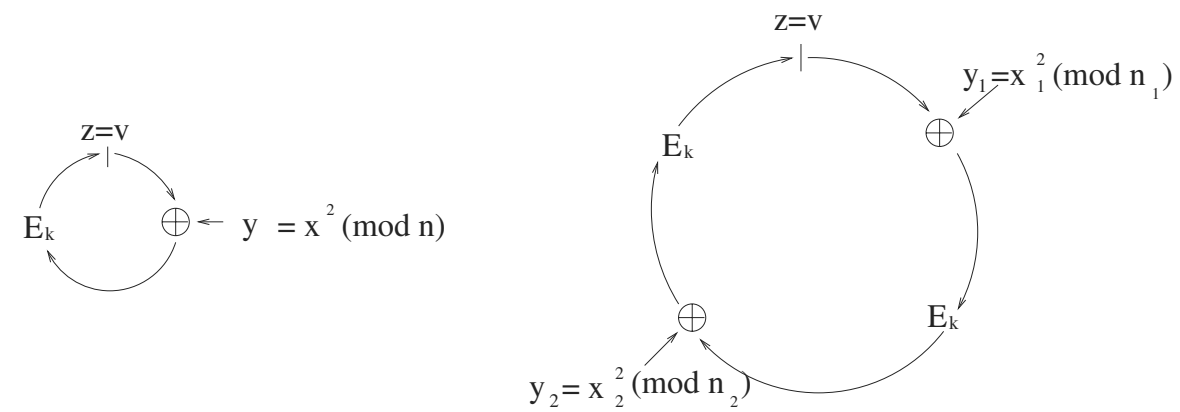

Fig. 3. Rabin-based Ring Signatures with $r=1,2$

In regular ring signatures it is provably impossible for an adversary to expose the signer's identity. However, there may be cases in which the signer himself 
wants to have the option of later proving his authorship of the anonymized email (e.g., if he is successful in toppling the disgraced Prime Minister). Yet another possibility is that the signer $A$ wants to initially use $\{A, B, C\}$ as the list of possible signers, but later prove that $\mathrm{C}$ is not the real signer. There is a simple way to implement these options, by choosing the $x_{i}$ values for the nonsigners in a pseudorandom rather than truly random way. To show that $\mathrm{C}$ is not the author, A publishes the seed which pseudorandomly generated the part of the signature associated with $\mathrm{C}$. To prove that A is the signer, A can reveal a single seed which was used to generate all the nonsigners' parts of the signature. The signer A cannot misuse this technique to prove that he is not the signer since his part is computed rather than generated, and is extremely unlikely to have a corresponding seed. Note that these modified versions can guarantee only computational anonymity, since a powerful adversary can search for such proofs of nonauthorship and use them to expose the signer.

\section{References}

1. Jan Camenisch. Efficient and generalized group signatures. In Walter Fumy, editor, Advances in Cryptology - Eurocrypt '97, pages 465-479, Berlin, 1997. Springer. Lecture Notes in Computer Science 1233.

2. David Chaum and Eugène Van Heyst. Group signatures. In D.W. Davies, editor, Advances in Cryptology - Eurocrypt '91, pages 257-265, Berlin, 1991. SpringerVerlag. Lecture Notes in Computer Science No. 547.

3. Ronald Cramer, Ivan Damgård, and Berry Schoenmakers. Proofs of partial knowledge and simplified design of witness hiding protocols. In Yvo Desmedt, editor, Advances in Cryptology - CRYPTO '94, pages 174-187, Berlin, 1994. SpringerVerlag. Lecture Notes in Computer Science Volume 839.

4. W. Diffie and M. E. Hellman. New directions in cryptography. IEEE Trans. Inform. Theory, IT-22:644-654, November 1976.

5. G. H. Hardy and E. M. Wright. An Introduction to the Theory of Numbers. Oxford, fifth edition, 1979.

6. M. Jakobsson, K. Sako, and R. Impagliazzo. Designated verifier proofs and their applications. In Ueli Maurer, editor, Advances in Cryptology - EuroCrypt '96, pages 143-154, Berlin, 1996. Springer-Verlag. Lecture Notes in Computer Science Volume 1070.

7. M. Luby and C. Rackoff. How to construct pseudorandom permutations from pseudorandom functions. SIAM J. Computing, 17(2):373-386, April 1988.

8. M. Rabin. Digitalized signatures as intractable as factorization. Technical Report MIT/LCS/TR-212, MIT Laboratory for Computer Science, January 1979.

9. Ronald L. Rivest, Adi Shamir, and Leonard M. Adleman. A method for obtaining digital signatures and public-key cryptosystems. Communications of the ACM, 21(2):120-126, 1978.

10. Alfredo De Santis, Giovanni Di Crescenzo, Giuseppe Persiano, and Moti Yung. On monotone formula closure of SZK. In Proc. 35th FOCS, pages 454-465. IEEE, 1994. 